\title{
An episome-based gene expression modulation platform in the model diatom
}

Phaeodactylum tricornutum

Tessema K. Kassaw, Andrew J. Paton, Graham Peers*

Department of Biology, Colorado State University, Fort Collins, CO 80523, USA

*Corresponding author email: graham.peers@colostate.edu 
Table S1. Sequence information of all the genetic components used in this study. Endogenous constitutive promoters are PCR-amplified from Phaeodactylum tricornutum genomic DNA.

\begin{tabular}{|c|c|c|}
\hline Name & Type & Sequence \\
\hline DiaNEV & $\begin{array}{l}\text { Synthetic } \\
\text { Transcription } \\
\text { Factor }\end{array}$ & $\begin{array}{l}\text { ATGGCCCAGGCTGCCCTCGAACCCGGAGAAAAGCCCTACGCTTGCCCCGAATGTGGA } \\
\text { AAGTCCTTCTCGCAATCCTCCAACTTGGTTCGTCACCAGCGTACGCACACCGGAGAAA } \\
\text { AGCCGTATAAGTGCCCGGATGCGGTAAGTCCTTTCGCAGTCCTCGTCCCTCGTCCG } \\
\text { CCACCAACGCACCCACACCGGTGAGAAGCCCTACAAGTGTCCGGAGTGCGGTAAGTC } \\
\text { GTTTTCGCGCTCCGATAAGCTCGTGCGCCACCAGCGCACGCACACGGGTAAGAAGAC } \\
\text { CTCGGGACAGGCCGGACGTCGTAACGAAATGGGAGCTTCCGGAGACATGCGTGCTGC } \\
\text { CAACTTGTGGCCCTCCCCGCTCGTCATTAAGCACACCAAGAAGAACTCCCCGCCTTG } \\
\text { TCCTTGACCGCTGACCAGATGGTCTCCGCCTTGTTGGATGCCGAACCGCCCATGATCT } \\
\text { ACTCCGAATACGATCCTCCCGTCCCTCTCCGAAGCCTCCATGATGGGTTTGTTGACC } \\
\text { AACCTCGCTGATCGTGAACTCGTTCACATGATTAACTGGGCCAAGCGTGTCCCCGGAT } \\
\text { TTGGAGACTTGAACCTCCACGATCAGGTCCACCTCCTCGAGTGCGCTTGGCTCGAAAT } \\
\text { CTTGATGATTGGTCTCGTCTGGCGTTCCATGGAACACCCCGGTAAGTTGTTGTTCGCTC } \\
\text { CCAACTTGCTCTTGGACCGTAATCAGGGTAAGTGTGTCGAAGGAATGGTCGAAATCTT } \\
\text { CGACATGTTGCTCGCTACCTCCTCGCGTTTCCGTATGATGAACCTCCAGGGTGAAGAA } \\
\text { TTTGTCTGCCTCAAGTCCATTATTTTGCTCAACTCCGGAGTCTACACCTTCCTCTCCTCC } \\
\text { ACCTTGAAGTCCCTCGAAGAAAAGGACCACATCCACCGTGTCCTCGACAAGATTACC } \\
\text { GACACCTTGATTCACCTCATGGCCAAGGCTGGATTGACCCTCCAGCAGCAGCACCGTC } \\
\text { GCCTCGCCCAGCTCTTGCTCATTCTCTCCCACATCCGTCACATGTCCAACAAGGGAAT } \\
\text { GGAACACCTCTACAACATGAAGTGCAAGAACGTTGTCCCCCTCTATGACCTCCTCTTG } \\
\text { GAAATGTTGGATGCCCACCGCCTCCACGCCCCCGCCTCCCGTATGGGAGTCCCCCGG } \\
\text { AAGAACCCTCCCAGACCCAGTTGGCACCACCTCGTCCACCTCCGCTCACTCCTTGCA } \\
\text { GACCTACTACATTCCCCCGGAAGCTGAAGGATTCCCCAACACCATTGGACGCGCCGAC } \\
\text { GCCCTCGACGATTTTGACCTCGATATGCTCGGATCGGATGCCCTCGATGACTTCGACT } \\
\text { TGGACATGTTGGGATCGGACGCCTTGGATGATTCGATCTCGACATGCTCGGTTCCGA } \\
\text { CGCTTTGGACGACTTCGACCTCGATATGTTGATTAACTACCCCTACGACGTCCCCGAC } \\
\text { TACGCTTCCTGA }\end{array}$ \\
\hline DiaXVE & $\begin{array}{l}\text { Synthetic } \\
\text { Transcription } \\
\text { Factor }\end{array}$ & $\begin{array}{l}\text { ATGAAGGCCTTGACCGCTCGTCAGCAGGAAGTTTTTGATTTGATTCGTGACCACATTT } \\
\text { CCCAGACCGGAATGCCCCCGACCCGTGCCGAAATTGCTCAGCGCCTCGGATTCCGTTC } \\
\text { CCCCAATGCTGCCGAAGAACACCTCAAGGCCCTCGCTCGTAAGGGAGTTATTGAAAT } \\
\text { GTCTCCGGAGCCTCCCGCGGTATTCGTTTGCTCCAGGAAGAAGAAGAAGGACTCCCCT } \\
\text { TGGTTGGACGTGTTGCCGCTGGAGAACCCTCCTCGGCCCCGCCCACCGATGTCTCCCT } \\
\text { CGGAGACGAATTCACTTGGATGGAGAAGACGTCGCCATGGCTCACGCTGATGCCTT } \\
\text { GGACGACTTCGATCTCGACATGCTCGGAGACGGAGATTCCCGGGACCGGATCAC } \\
\text { CCCGCACGATTCCGCTCCCTATGGAGCCTTGGACATGGCTGACTTCGAATTTGAACAG } \\
\text { ATGTTCACCGACGCCTTGGGAATTGATGAATATGGAGGAGACCCCTCCGCCGGAGAC } \\
\text { ATGCGTGCTGCCAACTTGTGGCCCTCGCCCTTGATGATTAAGCGTTCCAAGAAGAACT } \\
\text { CCCTCGCTCTCTCGTTGACCGCTGACCAGATGGTTCCGCCCTCTTGGACGCCGAACC } \\
\text { GCCGATTTTGTACTCCGAATACGATCCCACCCGTCCCTTCTCCGAAGCCTCGATGATG } \\
\text { GGACTCCTCACCAACCTCGCTGATCGTGAACTCGTCCACATGATTAACTGGGCCAAGC } \\
\text { GTGTTCCGGGATTCGTCATCTCACCTTGCACGACCAAGTCACCTCTTGGAATGCGC } \\
\text { TTGGCTCGAAATTTGATGATTGGACTCGTCTGGCGTTCCATGGAACACCCCGTTAAG } \\
\text { TTGCTCTTCGCCCCGAACCTCTTGCTCGATCGTAACCAGGGAAAGTGTGTTGAAGGAA } \\
\text { TGGTCGAAATTTCGACATGCTCTTGGCCACCTCCTCGCGCTTTCGTATGATGAATTG } \\
\text { CAGGGAGAAGAATTGTCTGTCTCAAGTCCATCATTCTCCTCAACTCCGGAGTTTATA } \\
\text { CCTTCCTCTCCTCGACCCTCAAGTCCTTGGAAGAAAAGGACCACATTCACCGTGTCCT } \\
\text { CGATAAGATTACCGACACCTTGATTCACCTCATGGCCAAGGCTGGACTCACCTTGCAG } \\
\text { CAGCAACACCAGCGTCTCGCCCAGCTCTTGTTGATTCTCTCCCACATTCGTCACATGTC } \\
\text { CAACAAGGGAATGGAACACCTCTACTCGATGAAGTGTAAGAACGTCGTTCCCCTCTAC } \\
\text { GACCTCTTGCTCGAAATGTTGGATGCTCACCGTCTCCACGCTCCCACCTCCCGTGGAG } \\
\text { GAGCCTCCGTCGAAGAAACCGACCAGTCCCACCTCGCCACCGCTGGATCGACCTCGTC } \\
\text { CCACTCCCTCCAGAAGTATTACATTACCGGAGAAGCCGAAGGATTCCCCGCTACCGTC } \\
\text { TGA }\end{array}$ \\
\hline
\end{tabular}




\begin{tabular}{|c|c|c|}
\hline DiaLhGR & $\begin{array}{l}\text { Synthetic } \\
\text { Transcription } \\
\text { Factor }\end{array}$ & 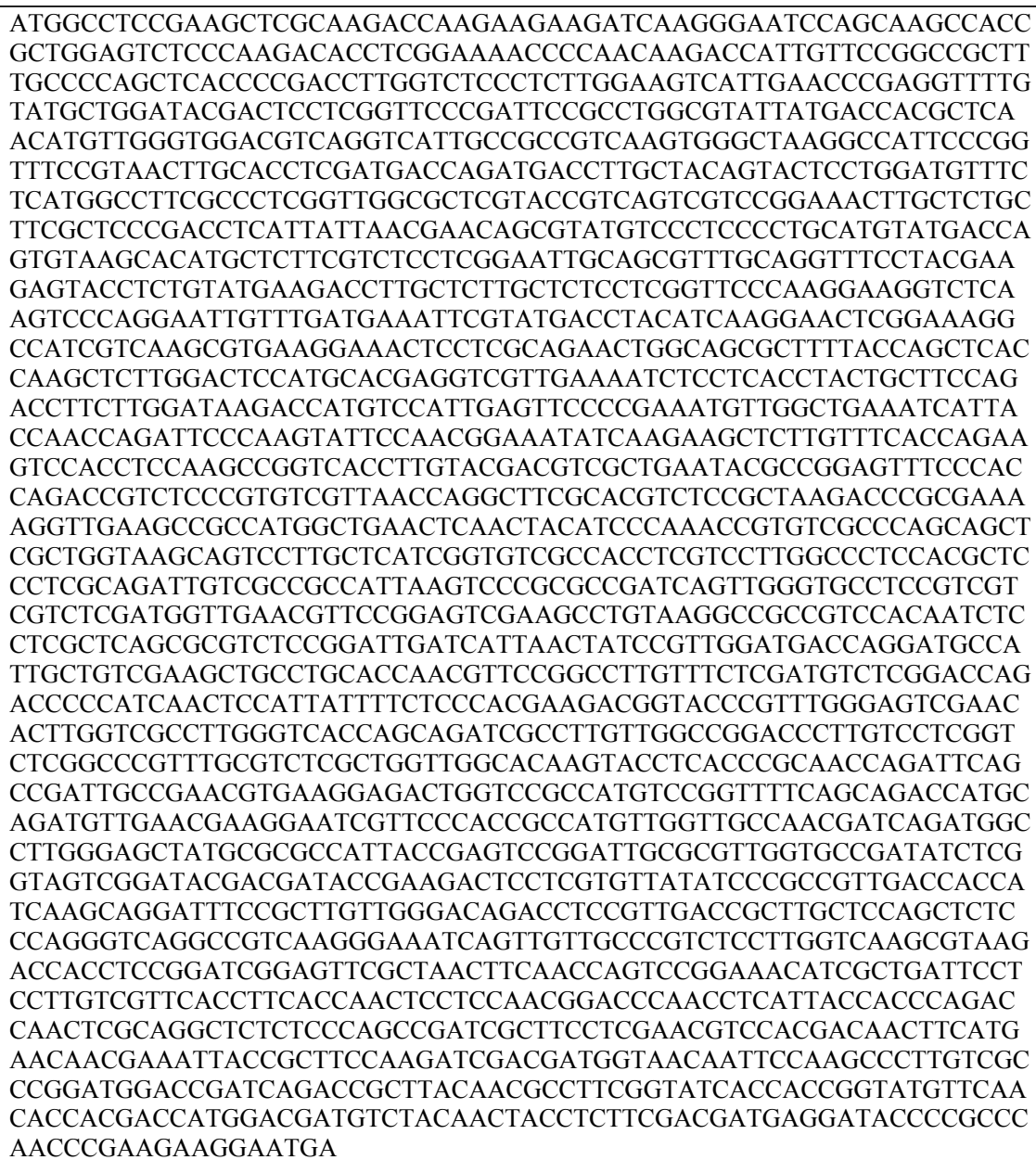 \\
\hline DiaAlcR & $\begin{array}{l}\text { Synthetic } \\
\text { Transcription } \\
\text { Factor }\end{array}$ & 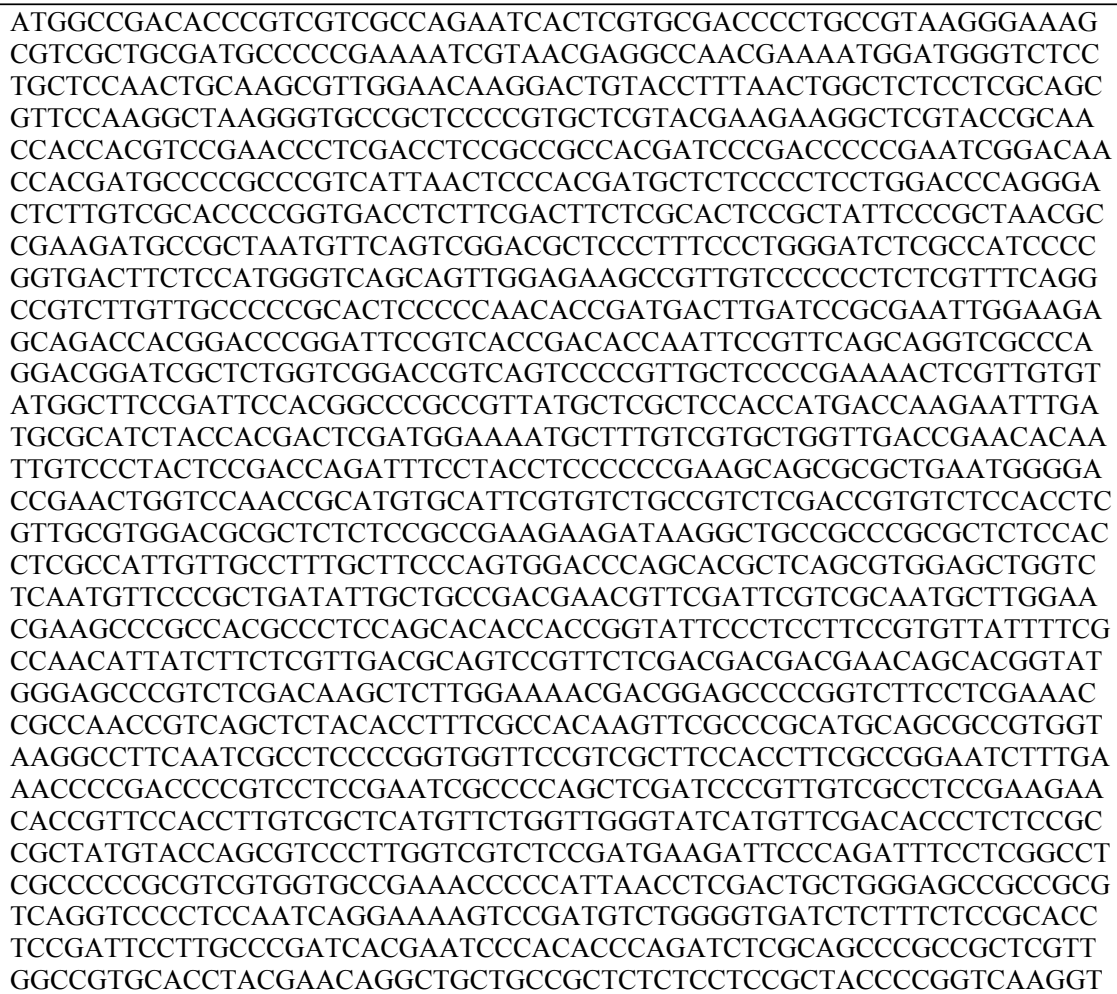 \\
\hline
\end{tabular}




\begin{tabular}{|c|c|c|}
\hline & & $\begin{array}{l}\text { TCTCTTGTACCGTCGCGTTACCCAGTTGCAGACCCTCTTGTACCGTGGAGCCTCCCCCG } \\
\text { CCCGTCTCGAGGCCGCCATTCAGCGTACCCTCTACGTCTACAATCACTGGACCGCCAA } \\
\text { GTACCAGCCCTTCATGCAGGACTGTGTCGCCAATCACGAACTCCTCCCCTCCCGTATT } \\
\text { CAGTCCTGGTACGTCATCCTCGACGGTCACTGGCACCTCGCTGCCATGCTCTTGGCCG } \\
\text { ATGTCCTCGAATCGATTGACCGCGATTCGTACTCCGATATCAATCACATTGATCTCGTT } \\
\text { ACCAAGCTCCGCTTGGACAACGCCTTGGCCGTCTCCGCCTTGGCCCGTTCGTCCCTCC } \\
\text { GTGGTCAGGAATTGGACCCGGAAAGCTTCGCCCATGTATCGCCACTTCCACGACTC } \\
\text { GTTGACCGAAGTTGCCTTTCTCGTCGAACCCTGGACGGTCGTCTTGATTCACTCCTTCG } \\
\text { CCAAGGCTGCCTACATTCTCCTCGACTGTTTGGACCTCGATGGACAGGGTAACGCCTT } \\
\text { GGCTGGATACCTCCAGTTGCGCCAGAACTGTAACTACTGCATTCGTGCCCTCCAGTTT } \\
\text { TTGGGACGTAAGTCCGACATGGCTGCCCTCGTCGCCAAGGACTTGGAGCGTGGACTCA } \\
\text { ACGGAAAGGTTGACTCCTTCTTGTAA }\end{array}$ \\
\hline DiarTetR & $\begin{array}{l}\text { Synthetic } \\
\text { Transcription } \\
\text { Factor }\end{array}$ & $\begin{array}{l}\text { ATGTCCCGTTTGGACAAGTCCAAGGTCATCAACGGAGCCTTGGAATTGCTCAACGGAG } \\
\text { TCGGAATCGAAGGATTGACCACCCGTAAGTTGGCCCAGAAGTTGGGAGTTGAACAGC } \\
\text { CCACCCTCTACTGGCACGTCAAGAACAAGCGTGCCTTGCTCGACGCCTTGCCCATCGA } \\
\text { GATGTTGGACCGTCACCACACCCACTTCTGCCCCTGGAAGGAGAATCCTGGCAGGAC } \\
\text { TTTTTGCGTAACAACGCCAAGTCCTACCGCTGCGCTCTCTTGTCCCACCGTGACGGAG } \\
\text { CTAAGGTCCACTTGGGAACCCGCCCCACCGAAAAGCAGTACGAGACCTTGGAAAACC } \\
\text { AGCTCGCCTTCTTGTGTCAGCAAGGATTCTCCTTGGAAAACGCCTTGTACGCTTTGTCC } \\
\text { GCCGTCGGACACTTTACCTTGGGATGCGTCTTGGAGGAACAGGAACACCAGGTCGCC } \\
\text { AAGGAGGAACGTGAAACCCCCACCACCGACTCCATGCCCCCGTTGCTCCGTCAGGCC } \\
\text { ATCGAATTGTTCGACCGCCAGGGAGCCGAACCCGCCTTCTTGTTCGGATTGGAACTCA } \\
\text { TCATCTGCGGATTGGAAAAGCAGTTGAAGTGCATCCGGAGGACCCACCGACGCTT } \\
\text { TGGACGATTTGATCTCGACATGTTGCCGGCTGATGCTCTCGATGACTTCGACTTGGA } \\
\text { CATGTTGCCGGCCGACGCCTTGGACGATTTCGATCTCGATATGTTGCCCGGATAA }\end{array}$ \\
\hline DiaDIG & $\begin{array}{l}\text { Synthetic } \\
\text { Transcription } \\
\text { Factor }\end{array}$ & $\begin{array}{l}\text { ATGAACAAGATCCCCATCAAGGACTTGTTGAACCCCCAGATTACCGATGAATTTAAGT } \\
\text { CCTCCATCTTGGACATTAACAAGAAGTTGTTCTCCATTTGTTGCAACCTCCCCAAGCTC } \\
\text { CCGGAATCCGTCACCACCGAAGAAGAAGTCGAATTGCGTGATATCTTGGGATTCTTGT } \\
\text { CCCGTGCCAACAAGAACCGTAAGATTATGAAGTTGTTGTCCTCCATTGAACAGGCCTG } \\
\text { CGACATTTGCCGTTGAAGAAGTGCAAGTGCTCCAAGGAAAAGCCCAATGCGCCAA } \\
\text { GTGCCTCAAGAACAACTGGGAATGCCGTTACTCCCCCAAGACCAAGCGTTCCCCCCTC } \\
\text { ACCCGTGCCCACCTCACCGAAGTCGAATCCCGTCTCGAACGTCTCGAACAGCTCTTCC } \\
\text { TCTTGATTTCCCCCGTGAAGACCTCGACATGATTCTCAAGATGGATTCCTTGCAGGAT } \\
\text { ATTAAGGCTCTCTTGGGAGGTTCCGGAGGTTCCATGAATGCTAAGGAAATGTTGTC } \\
\text { ACGCCCTCCGTTTGCTCGAAAATGGAGATGCCCGTGGATGGTCCGACCTCTTCCACCC } \\
\text { CGAAGGAGTCCTCGAATACCCCTACCCGCCCCCGGGATACAAGACCCGTTTCGAAGG } \\
\text { ACGTGAAACCATTTGGGCCCACATGCGTCTCTTCCCGAATACATGACCATCCGTTTC } \\
\text { ACCGACGTCCAGTTCTACGAAACCGCCGACCCCGACCTCGCCATTGGAGTCTTCCACG } \\
\text { GAGACGGAGTCCTCACCGCCTCCGGAGGAAAGCTCGCCTACGACTACATTGCCGTCTG } \\
\text { GCGTACCCGTGACGGACAGATTCTCCTCTACCGTCTCTTCTTCAACCCCCTCCGTGTCC } \\
\text { TCGAACCCCTCGGAGGAGTCGAAGCCGCTGCCAAGATTGTCCAGGGAGCCTCCGCCG } \\
\text { ACGGTTCCGCCTACTCCCGTGCCCGTACCAAGAACAACTACGGATCGACCATCGAAG } \\
\text { GACTCCTCGACTTGCCCGATGATGACGCTCCCGAAGAAGCCGGATTGGCCGCTCCCCG } \\
\text { TCTCTCCTTCTTGCCCGCCGGACACACCCGTCGTCTCTCCACCGCTCCGCCCACCGATG } \\
\text { TCTCCCTCGGAGACGAACTCCACTTGGACGGAGAAGACGTCGCCATGGCCCACGCCG } \\
\text { ACGCCCTCGACGATTCGACCTCGACATGTTGGGAGACGGAGATTCCCCCGGACCCGG } \\
\text { ATTCACCCCCACGACTCCGCCCCCACGGAGCCCTCGACATGGCCGACTTCGAATTT } \\
\text { GAACAGATGTTCACCGACGCCCTCGGAATTGACGAATACGGAGGATAA }\end{array}$ \\
\hline 10xN1 & $\begin{array}{l}\text { Inducible } \\
\text { Promoter }\end{array}$ & $\begin{array}{l}\text { GTCGACGGGGTAGAAAAAGGGGTAGAAAACCAAGGGGTAGAAAAAGGGGTAGAAAA } \\
\text { CCAAGGGGTAGAAAAAGGGGTAGAAAACCAAGGGGTAGAAAAAGGGGTAGAAAACC } \\
\text { AAGGGGTAGAAAAAGGGGTAGAAGCAAGACCCTTCCTCTATATAAGGAAGTTCATTT } \\
\text { CATTTGGAGAGG }\end{array}$ \\
\hline $\begin{array}{l}\text { LexA_min } \\
\text { 35S }\end{array}$ & $\begin{array}{l}\text { Inducible } \\
\text { Promoter }\end{array}$ & $\begin{array}{l}\text { AACCGGTCTTGCATCCAGCTGGGCTGCAGGTCGAGGCTAAAAAACTAATCGCATTATC } \\
\text { ATCCCCTCGACGTACTGTACATATAACCACTGGTTTTATATACAGCAGTACTGTACAT } \\
\text { ATAACCACTGGTTTTATATACAGCAGTCGACGTACTGTACATATAACCACTGGTTTTA } \\
\text { TATACAGCAGTACTGTACATATAACCACTGGTTTATATACAGCAGTCGAGGTAAGAT } \\
\text { TAGATATGGATATGTATATGGATATGTATATGGTGGTAATGCCATGTAATATGCTCGA } \\
\text { CTCTAGGATCTTCGCAAGACCCTTCCTCTATATAAGGAAGTTCATTTCATTTGGAGAG } \\
\text { GACACGCTGAAGCTAGTCGACTCTAGCCAAA }\end{array}$ \\
\hline pOp6 & $\begin{array}{l}\text { Inducible } \\
\text { Promoter }\end{array}$ & $\begin{array}{l}\text { GACAATTGTGAGCGCTCACAATTGAAAGACTAGAAAGAAGAAAGGGAAGAGAAAGA } \\
\text { ATTGTGAGCGCTCACAATTGAAAGACTAGAAAGAAGAAAGGGAAGAGAAAGAATTG } \\
\text { TGAGCGCTCACAATTGAAAGACTAGAAAGAAGAAAGGGAAGAGAAAGAATTGTGAG } \\
\text { CGCTCACAATTGAAAGACTAGAAAGAAGAAAGGGAAGAGAAAGAATTGTGAGCGCT } \\
\text { CACAATTGAAAGACTAGAAAGAAGAAAGGGAAGAGAAAGAATTGTGAGCGCTCACA } \\
\text { ATTGAAAGACTAGTGGATCGATCTTCGCAAGACCCTTCCTCTATATAAGGAAGTTCAT } \\
\text { TTCATTTGGAGAGGA }\end{array}$ \\
\hline
\end{tabular}




\begin{tabular}{|c|c|c|}
\hline pAlcA & $\begin{array}{l}\text { Inducible } \\
\text { Promoter }\end{array}$ & $\begin{array}{l}\text { GTCGACTCGATAGTTGTGATAGTTCCCACTTGTCCGTCCGCATCGGCATCCGCAGCTC } \\
\text { GGGATAGTTCCGACCTAGGATTGGATGCATGCGGAACCGCACGAGGGCGGGGCGGAA } \\
\text { ATTGACACACCACTCCTCTCCACGCACCGTTCAAGAGGTACGCGTATAGAGCCGTATA } \\
\text { GAGCAGAGACGGAGCACTTTCTGGTACTGTCCGCACGGGATGTCCGCACGGAGAGCC } \\
\text { ACAAACGAGCGGGGCCCCGTACGTGCTCTCCTACCCCAGGATCGCATCCCCGCATAGC } \\
\text { TGAACATCTATATAAGGAAGTTCATTTCATTTGGAGAGGACGACT }\end{array}$ \\
\hline pTRE & $\begin{array}{l}\text { Inducible } \\
\text { Promoter }\end{array}$ & $\begin{array}{l}\text { GTCGACGAGTTTACTCCCTATCAGTGATAGAGAACGTATGTCGAGTTTACTCCCTATC } \\
\text { AGTGATAGAGAACGATGTCGAGTTTACTCCCTATCAGTGATAGAGAACGTATGTCGA } \\
\text { GTTTACTCCCTATCAGTGATAGAGAACGTATGTCGAGTTTACTCCCTATCAGTGATAG } \\
\text { AGAACGTATGTCGAGTTTATCCCTATCAGTGATAGAGAACGTATGTCGAGTTTACTCC } \\
\text { CTATCAGTGATAGAGAACGTATGTCGAGGCAAGACCCTTCCTCTATATAAGGAAGTTC } \\
\text { ATTTCATTTGGAGAGGA }\end{array}$ \\
\hline pUAS & $\begin{array}{l}\text { Inducible } \\
\text { Promoter }\end{array}$ & $\begin{array}{l}\text { GTCGACTCGACCCTTATGACGCACATCCCACTATGCCTTAGCGGATTAGAAGCCGCCG } \\
\text { AGTGGGTGACAGCCCTCCGAGCGGGCCGCACTGCTCCGAGCGGAGGACTGTCCTCCG } \\
\text { AGCGCAAGACCTTCCTCTATATAAGGAAGTTCATTTCATTTGGAGAGGAAGCTCTCAA } \\
\text { TCCAAATAATCTGCAGATCCTAGACGATCGTTTCGC }\end{array}$ \\
\hline DIAeYFP & $\begin{array}{l}\text { Reporter } \\
\text { Protein }\end{array}$ & $\begin{array}{l}\text { ATGGTGAGCAAGGGCGAGGAGCTGTTCACCGGGGTGGTGCCCATCCTGGTCGAGCTG } \\
\text { GACGGCGACGTAAACGGCCACAAGTTCAGCGTGTCCGGCGAGGGCGAGGGCGATGCC } \\
\text { ACCTACGGCAAGCTGACCCTGAAGTTCATCTGCACCACCGGCAAGCTGCCCGTGCCCT } \\
\text { GGCCCACCCTCGTGACCACCTTCGGCTACGGCCTGCAGTGCTTCGCCCGCTACCCCGA } \\
\text { CCACATGAAGCAGCACGACTTCTTCAAGTCCGCCATGCCCGAAGGCTACGTCCAGGA } \\
\text { GCGCACCATCTTCTTCAAGGACGACGGCAACTACAAGACCCGCGCCGAGGTGAAGTT } \\
\text { CGAGGGCGACACCCTGGTAACCGCATCGAGCTGAAGGGCATCGACTTCAAGGAGGA } \\
\text { CGGCAACATCCTGGGGCACAAGCTGGAGTACAACTACAACAGCCACAACGTCTATAT } \\
\text { CATGGCCGACAAGCAGAAGAACGGCATCAAGGTGAACTTCAAGATCCGCCACAACAT } \\
\text { CGAGGACGGCAGCGTGCAGCTCGCCGACCACTACCAGCAGAACACCCCCATCGGCGA } \\
\text { CGGCCCCGTGCTGCTGCCCGACAACCACTACCTGAGCTACCAGTCCGCCCTGAGCAAA } \\
\text { GACCCCAACGAGAAGCGCGATCACATGGTCCTGCTGGAGTTCGTGACCGCCGCCGGG } \\
\text { ATCACTCTCGGCATGGACGAGCTGTACAAGTGA }\end{array}$ \\
\hline NOSt & Terminator & $\begin{array}{l}\text { CGTTCAAACATTTGGCAATAAAGTTTCTTAAGATTGAATCCTGTTGCCGGTCTTGCGAT } \\
\text { GATTATCATATAATTTCTGTTGAATTACGTTAAGCATGTAATAATTAACATGTAATGC } \\
\text { ATGACGTTATTTATGAGATGGGTTTTTATGATTAGAGTCCCGCAATTATACATTTAATA } \\
\text { CGCGATAGAAAACAAAATATAGCGCGCAAACTAGGATAAATTATCGCGCGCGGTGTC } \\
\text { ATCTATGTTACTAGATCGGG }\end{array}$ \\
\hline NRt & Terminator & $\begin{array}{l}\text { CAATTTACTTTAAGACTAGCGCAATTTTTTTCTTACATTAGATGAACATAAAACGACG } \\
\text { ATGAGGATCACTGTTAGGGAAACGATGAACTACGCTGAGGACTTCCTATTATTCGTCC } \\
\text { TTTGATGGCGTGGTTGTTCTTTGGTTTGCGGCCGAGCGCAACGACCAAGGCCTGTACC } \\
\text { AAAAACGAAGTTGATAATCAGATATTTGAGTTGTAACAAGAAACTAAAGCATTATTTT } \\
\text { AGCAGTAGCACGGATCAGGATCTAAAATGAACGCATCCGGA }\end{array}$ \\
\hline p35S & $\begin{array}{l}\text { Constitutive } \\
\text { Promoter }\end{array}$ & $\begin{array}{l}\text { CATGGAGTCAAAGATTCAAATAGAGGACCTAACAGAACTCGCCGTAAAGACTGGCGA } \\
\text { ACAGTTCATACAGAGTCTCTTACGACTCAATGACAAGAAGAAAATCTTCGTCAACATG } \\
\text { GTGGAGCACGACACACTTGTCTACTCCAAAAATATCAAAGATACAGTCTCAGAAGAC } \\
\text { CAAAGGGCAATTGAGACTTTTCAACAAAGGGTAATATCCGGAAACCTCCTCGGATTCC } \\
\text { ATTGCCCAGCTATCTGTCACTTTATTGTGAAGATAGTGGAAAAGGAAGGTGGCTCCTA } \\
\text { CAAATGCCATCATTGCGATAAAGGAAAGGCCATCGTTGAAGATGCCTCTGCCGACAG } \\
\text { TGGTCCCAAAGATGGACCCCACCCACGAGGAGCATCGTGGAAAAAGAAGACGTTCC } \\
\text { AACCACGTCTTCAAAGCAAGTGGATTGATGTGATATCTCCACTGACGTAAGGGATGAC } \\
\text { GCACAATCCCACTATCCTTCGCAAGACCCTTCCTCTATATAAGGAAGTTCATTTCATTT } \\
\text { GGAGAGAACACGGGGGACT }\end{array}$ \\
\hline pNR & $\begin{array}{l}\text { Constitutive } \\
\text { Promoter }\end{array}$ & $\begin{array}{l}\text { CATATGCGGAAGTGACTGTAAACGAGAAGTGCACGAAGCCTTTTCTTGTGACGTCACA } \\
\text { AACCGAACAGCCCTACGTGGCGTGCGACTTTCCGGCACTTTGGATATCGTTGCCCTAT } \\
\text { ATGTATTGGGTGTATACATGTCGTATTCCAACTACGACAGAAAACAGTCAGTTACGGG } \\
\text { TATTCGAACTTCCGGCACCTGCAGCGAGGAGTTTTGTGTCCGTCGCACATCCTCCGTG } \\
\text { CTTTCGGCACCTACGTAGGCGGCAAACTTCCTGCCTTCCGCCCGCCTTGCGGCATTCC } \\
\text { GGCTCCGGGCCAGAATTGCCCGGGTGTTCACAATTTGCCTCCTCACGAAAAAACGTT } \\
\text { CTACTTTGTATTTTGGTCGGGTTTCGGATCCTTCCAGCAACCATTCTCATTCAAAGTCA } \\
\text { CCACTTGTGCGAACG }\end{array}$ \\
\hline pEF2 & $\begin{array}{l}\text { Constitutive } \\
\text { Promoter }\end{array}$ & $\begin{array}{l}\text { TCCATTTTGACATGTTTCCTAGCTAGAAGGAACAGCTATTCATATGAATTTTCCGAGGT } \\
\text { CACGCTGTGATGGATCGATTCGTTCCCGTCACAGACGCAAGCAGCGAGGTGATGGCT } \\
\text { GGACGGGCACGCGTCACAATTTAGAACAACCCGGACTCTTGTGACGCGAGCTCCGGG } \\
\text { TGCGGAAAACCTACGGGGTGAAAGGAGCCGCAGCTTCCACCCATTACTTGACTGAT } \\
\text { AACCCAGTACACACAACGCTACCGTGACGGAAATGCCGCGCGCTGTGAAGCCGTGGT } \\
\text { GAATCTTTCGCTGCTGCTCGTTCTCTCCACACACAGAATTGCACGGCTTGACGATTAG } \\
\text { CAAAAAGCAGAAGAAGCAGTGTCAAGATGGTGAGTGTCTTGAGTACAAAAGTAGCGC } \\
\text { ACGGTAGTGCAGAATACGGCTGTGTAGACTATGCGGCGTCGGAGTAGCCGAGATCCC } \\
\text { GTAAAAAACGGTAGGAATCGGAGGAATCTCAATGAGTGATCGTGCTGCGTACGCCGC } \\
\text { TTCCGTTGGGGACCCTACGATCGCGTAGTTCTTGTTAGCAACGACATGCCTTTACGTC } \\
\text { GCGAAGGTCTTTCAAGTAACTAAAACAACGGAATATGTCAAGATTACCTCGATTGAT } \\
\text { GGATTCGAATGAGGATCGGGATCAGGAGTTCTATTGACACTAGTTGATTTCCTCAATG }\end{array}$ \\
\hline
\end{tabular}




\begin{tabular}{|c|c|c|}
\hline & & $\begin{array}{l}\text { CGAATGCCGCTCAATGTCCCCCTTTCTCATCTATGACTGTTTACTTCCCTTAGGTGAAC } \\
\text { TTTACAGTTGAACAG }\end{array}$ \\
\hline pCIP1 & $\begin{array}{l}\text { Constitutive } \\
\text { Promoter }\end{array}$ & $\begin{array}{l}\text { TACGTAGAATCCTACGTAAAATGGACGAAAAGGAATAATATTAACTTAATCCTTTTCG } \\
\text { TCCATTTTATAGTGTGACATGTTGAGTCCCGCCTGTTATTAACCAATACAGCCCCCATC } \\
\text { CCATAATGCGGCCTGAGGGCCTATCATCTTGCGCTGGTAGCGCGCTTTCTTTCTGTTTT } \\
\text { GGCAGGCGAGTATTGCCCTGGCAGGCGGCGTCCTACGGCAGGCGCTCGTGGTCAATA } \\
\text { CAACATGTCCATTATACCAACGTAAGATTCTGATACTAACGCGATTTTTTGGTCTCAA } \\
\text { ACAAGTATGGGGGTGGTAACCAACCCCAATGCAAAAATGCATCACGAACACAAAGGA } \\
\text { TGTTCCAATTAACCTTTATGTAACGGACTTCTTGTTTTCAATCGTTCCAAAACTCACCA } \\
\text { ATGTACAGATGCGAAAAAAAGATCAGTCAGATCATAAACAACTGCCTTCCTTTTTTTC } \\
\text { ATAAAAATTGTAATGAGCTACAACACGTACATTCGCA }\end{array}$ \\
\hline pH4 & $\begin{array}{l}\text { Constitutive } \\
\text { Promoter }\end{array}$ & $\begin{array}{l}\text { TTTGACGGTTTTTTTTACAAGAGAAGAGTTCTTGAAATTTGTGAGGTTAAAGTGTGTG } \\
\text { GCTTCCGCCGTAGTCAAGGAGCGTGCGGTTGCCGATCGCACCGGTACGTTCTGTAGAA } \\
\text { ATGAACACAGTGTGTTGAATTGAAAGTATGGCGCAGGTATGGTTGTGATAAGTAGCA } \\
\text { GCCGCGCCGAGACAAACAAACTTTGGTTTCTACGACAATCTCTGTAGACAAGTACTAG } \\
\text { AAACCCGTTTGAACGAGCATAAATCTGCACCGGCAGGCCACCAGACATCGTTTCAAC } \\
\text { GTAATATTCTACGTAACCATTTTATCCCAGGAAACCTACGGCCTGTGAACCACCGAGA } \\
\text { CGGAGCACTCACAATTCGCTCTCGGCAACAACCGACAATCGTCTTACTCACAGTCAAT } \\
\text { ACCGAAAACAAACAACAGCCAAC }\end{array}$ \\
\hline pRSV & $\begin{array}{l}\text { Constitutive } \\
\text { Promoter }\end{array}$ & $\begin{array}{l}\text { GCGATGTACGGGCCAGATATACGCGTATCTGAGGGGACTAGGGTGTGTTTAGGCGAA } \\
\text { AAGCGGGGCTTCGGTTGTACGCGGTTAGGAGTCCCCTCAGGATATAGTAGTTTCGCTT } \\
\text { TTGCATAGGGAGGGGGAAATGTAGTCTTATGCAATACTCTTGTAGTCTTGCAACATGG } \\
\text { TAACGATGAGTTAGCAACATGCCTTACAAGGAGAGAAAAAGCACCGTGCATGCCGAT } \\
\text { TGGTGGAAGTAAGGTGGTACGATCGTGCCTTATTAGGAAGGCAACAGACGGGTCTGA } \\
\text { CATGGATTGGACGAACCACTGAATTCCGCATTGCAGAGATATTGTATTTAAGTGCCTA } \\
\text { GCTCGATACAATAAACGCCATTTGACCATTCACCACATTGGTGTGCACCTCC }\end{array}$ \\
\hline pCMVEM & $\begin{array}{l}\text { Constitutive } \\
\text { Promoter }\end{array}$ & $\begin{array}{l}\text { CGTTACATAACTTACGGTAAATGGCCCGCCTGGCTGACCGCCCAACGACCCCCGCCCA } \\
\text { TTGACGTCAATAATGACGTATGTTCCCATAGTAACGCCAATAGGGACTTTCCATTGAC } \\
\text { GTCAATGGGTGGAGTATTTACGGTAAACTGCCCACTTGGCAGTACATCAAGTGTATCA } \\
\text { TATGCCAAGTACGCCCCCTATTGACGTCAATGACGGTAAATGGCCCGCCTGGCATTAT } \\
\text { GCCCAGTACATGACCTTATGGGACTTTCCTACTTGGCAGTACATCTACGTATTAGTCAT } \\
\text { CGCTATTACCATGGTGATGCGGTTTGGCAGTACATCAATGGGCGTGGATAGCGGTTT } \\
\text { GACTCACGGGGATTTCCAAGTCTCCACCCCATTGACGTCAATGGGAGTTTGTTTGGC } \\
\text { ACCAAAATCAACGGGACTTTCCAAAATGTCGTAACAACTCCGCCCCATTGACGCAAAT } \\
\text { GGGCGGTAGGCGTGTACGGTGGGAGGTCTATATAAGCAGAGCTAAGCTCCCCTGTT } \\
\text { GACAATTAATCATCGGCATAGTATATCGGCATAGTATAATACGACAAGGTGAGGAAC } \\
\text { TAAACC }\end{array}$ \\
\hline pPt211 & $\begin{array}{l}\text { Constitutive } \\
\text { Promoter }\end{array}$ & $\begin{array}{l}\text { CATCTTGTTTCTCGTAAGCAAAATTTGTATGGAAAATTAGATCATGAGGCTACAACTC } \\
\text { AAGGGCATCAAATCAGGCTAGGATTGTTGTTATCACTGTCCAATTCATCTTCAAGCAA } \\
\text { AATCTCGACGACAGCATTCTCAGTAACTTGAAAACTTGCTGATTGCAATATAGCTCTG } \\
\text { ACCTTGAAGTTTATTGCTGCTGTACCAGGAAGGTTCAACTGGTTTCCACTTGGACGTC } \\
\text { ACACTTATATTGTGGCAGCTGCTTGACTGTGAATGATTTGCATTGCTGTAATGAATCC } \\
\text { ATTCATTGGCAGTATTTGTGATAGGACTTGCTTTCTTCGATACTAGCTAGCCCATTA } \\
\text { CGAATCTTCAAAACTATGCAGTTTACTAAAACTCGTGATATATTTGTCAAGGGACGAA } \\
\text { TTATTTTTAAGTCGTTTTTCTTATATAGAAGACCACCTGTTTAGTGGATGTGACAGGTC } \\
\text { TGAGCACCCATTTAGTGAATTTCCTATAAATATTTTGATTCCTTGGACTTGATTTTAGG } \\
\text { AGGCCACAAAAAGCCGCATATTTGGCGGCATAAAACGACAGAGCATGACCGCCCATC } \\
\text { TGTATTCCGGTTCTGTCGACCACAGAATGCGTTATTTTTGACTGCGACATCATAGATTG } \\
\text { CTTCACAATCACTTTCACAGCCAATATTACCCACTGATCGAGAGCATTCTTGAGATTC } \\
\text { CTTATATTTGACATTTCCACACGACCTTGGTTCACAAGAATGTCGCATGCGGAACT } \\
\text { GACTGTGACTGTGAGATGAAAGAAACGAATCATGAGCGTCAAGAATATGCGAACAGT } \\
\text { AATCTGGACTTGACATTCGAACTTGTTCTCCGGCTGCTTGACATCACCAAGAAAACAG } \\
\text { ACTTTGCGCTTCCGAAGTTACGAGTCGGTTTGCAGCCCTCCTACTGCTATGAGATATG } \\
\text { AGAACCCATATCCCTTACTCTCCCTCCTTTTCCATCATAAAATCTCTTGACAGCCAAA } \\
\text { TCAAATCAAATCGAACACAACAAATATAGC }\end{array}$ \\
\hline pPt202a & $\begin{array}{l}\text { Constitutive } \\
\text { Promoter }\end{array}$ & $\begin{array}{l}\text { ACGAATTTCCAACGTCCTAACTCCAATTTATGGTTTCAAACAATCCGAATTCGTTGGTC } \\
\text { CACAGTCAAGGAATTCGCAAGAATCCCTATTTCACTAGGCGTGCACTATGGGGTCGCT } \\
\text { TTTGAGGTTCTCATTTGCGGCGATCGTTAAAACGATTACAGAAATGCTACACTTCGCT } \\
\text { CTTTATATATATTACTCATTGAGCATCAGGAATGTCCAATGTCGTAACTCCGATTCATG } \\
\text { GTTTCAAACAATCCGAATTCGTTGGTCCGAGGAATCCGCAAAAAGTAATTGTAGTCTA } \\
\text { TGTACGACCGAGTCGCTGGTGAGGGTTTTTGTCGTTGGATTGCTCGAGCGAATTCTC } \\
\text { CGAGATTGTACAGAAAGCTCAACTTTCGGAAGGCATCTATGTGGTCCAACCAATCTAT } \\
\text { TCAAATATATCAATTGTTAAGAGCAGAGTTCGATCCTCACTATGTGGAGGGGCATGGT } \\
\text { GAGATTACGGAGTGCCGAGACATAAGAGCTCCCCACAATCAAGTTGATGGACATGCT } \\
\text { TCGAAAATGCCATGACTGAGAACGAGAACGCCCAGATGCTCCACGGCACGAGAACTC } \\
\text { GATGAACACGAACCGAATCCAACGCCAAAAACGCGTTGTCCAAAACATTGCAACTCA } \\
\text { CTCTCTCTCTCATAAAAACCAAAACGCCGCAAATTGCACCAGCTTTTTTGGTTGTCCTC } \\
\text { CGCCTCTTTCCGCACCACC }\end{array}$ \\
\hline
\end{tabular}




\begin{tabular}{|c|c|c|}
\hline pPt202 & $\begin{array}{l}\text { Constitutive } \\
\text { Promoter } \\
\text { (published } \\
\text { sequence) }{ }^{I}\end{array}$ & $\begin{array}{l}\text { ACGAATTTCCAACGTCCTAACTCCAATTTATGGTTTCAAACAATCCGAATTCGTTGGTC } \\
\text { CACAGTCAAGGAATTCGCAAGAATCACTATTTCACTACGCGTGCACTATGGGGTCGCT } \\
\text { TTTGTGGTTCTCATTTGCGGCGATCGTTAAAACGATTAAAGAAATGCTACACTTCGCT } \\
\text { CTTTATATATATTACTCATTGAGCATACGAATGTCCAATGTCGTAACTCCGATTCATGG } \\
\text { TTTCAAACAATCCGAATTCGTTGGTCCGAGGAATCCGCAAAAAGTAATTGTAGTCTAT } \\
\text { GTACGACCGAGTCGCTGGTGAGGGTTTTTTGCGTTGGATTGCTCGAGCGAATTCTCC } \\
\text { GAGATTGTACAGAAAGCTCAACTTTCGGAAGGCATCTATGTGGTCCAACCAATCTATT } \\
\text { CAAATATATCAATTGTTAAGAGCAGAGTCGATCCTCACTATGTGGAGGGGCATGGTG } \\
\text { AGATTACGGAGTGCCGAGACATAAGAGCTCCCCACAATCAAGTTGATGGACATGCTT } \\
\text { CGAAAATGCCATGACTGAGAACGAGAACGCCCAGATGCTCCACGGCACGAGAACTCG } \\
\text { ATGAACACGAACCGAATCCAACGCCAAAAACGCGTTGTCCAAAACATTGCAACTCAC } \\
\text { TCTCTCTCTCATAAAAACCAAAACGCCGCAAATTGCACCAGCTTTTTGGTTGTCCTCC } \\
\text { GCCTCTTTCCGCACCACC }\end{array}$ \\
\hline
\end{tabular}


Table S2. Primers used in this study.

\begin{tabular}{|c|c|c|c|}
\hline Primer & Orientation & Sequence (5' - 3') & Description \\
\hline P1 & F & ACGTTCTAGACATGGAGTCAAAGATTCA & \multirow{5}{*}{$\begin{array}{l}\text { Used to fix DIG inducible expression } \\
\text { module after synthesis }\end{array}$} \\
\hline $\mathrm{P} 2$ & $\mathrm{R}$ & ACGTGAATTCTTATCCTCCGTATTCGTCA & \\
\hline P3 & $\mathrm{F}$ & CGATGGATCCGTCGACTCGACCCTTATGACG & \\
\hline P4 & $\mathrm{R}$ & $\begin{array}{l}\text { CGATCCTGCAGGCCCGATCTAGTAACATAGATGACA } \\
\text { CC }\end{array}$ & \\
\hline P5 & F & TTACTCCCCCAAGACCAAGC & \\
\hline P6 & F & ACGTTCTAGACATATGCGGAAGTGACTGTAAA & \multirow[t]{2}{*}{ PCR amplification of NR Promoter } \\
\hline P7 & $\mathrm{R}$ & ACGTCCTAGGCGTTCGCACAAGTGGTGACT & \\
\hline P8 & F & ACGTGAGCTCCAATTTACTTTAAGACTAGCGCAATTT & \multirow[t]{2}{*}{ PCR amplification of NR terminator } \\
\hline P9 & $\mathrm{R}$ & ACGTCCTGCAGGTCCGGATGCGTTCATTTTA & \\
\hline $\mathrm{P} 10$ & F & ACTGTCTAGACATGGAGTCAAAGATTCAA & \multirow[t]{2}{*}{ PCR amplification of 35S Promoter } \\
\hline P11 & $\mathrm{R}$ & ACTGCCTAGGAGTCCCCCGTGTTCTCTCC & \\
\hline P26 & F & CGATTCTAGACATATGCGGAAGTGACTGTAAA & \multirow{2}{*}{$\begin{array}{l}\text { Used to replace } \mathrm{p} 35 \mathrm{~S} \text { by } \mathrm{pNR} \text { in the } \\
\text { inducible expression systems }\end{array}$} \\
\hline P27 & $\mathrm{R}$ & CGATGCGGCCGCCGTTCGCACAAGTGGTGACT & \\
\hline $\mathrm{P} 28$ & F & $\begin{array}{l}\text { CTCTAGATACGTAGAATCCTACGTAAAATGGACGAA } \\
\text { AAGGAATAATATTAACTTAATCCT }\end{array}$ & \multirow[t]{2}{*}{ PCR amplification of full ClP1 promoter } \\
\hline P29 & $\mathrm{R}$ & CGTACCTAGGTGCGAATGTACGTGTTGTAGC & \\
\hline $\mathrm{P} 30$ & F & CACAGGAGTCTGGACTTGACC & $\begin{array}{l}\text { Used to sequence verify all inducible } \\
\text { expression constructs }\end{array}$ \\
\hline P31 & F & CACCTCACCGAAGTCGAATC & \multirow{3}{*}{$\begin{array}{l}\text { Used to sequence verify digoxin } \\
\text { inducible expression cassette }\end{array}$} \\
\hline $\mathrm{P} 32$ & F & CTCGGAATTGACGAATACGG & \\
\hline $\mathrm{P} 33$ & F & CGACCACTACCAGCAGAACA & \\
\hline P34 & F & AGATGGTCTCCGCCTTGTT & \multirow{3}{*}{$\begin{array}{l}\text { Used to sequence verify 4-OHT } \\
\text { inducible expression cassette }\end{array}$} \\
\hline P35 & $\mathrm{F}$ & CCTACGACGTCCCCGACTAC & \\
\hline P36 & F & ACTTCAAGATCCGCCACAAC & \\
\hline P37 & F & CTGATGCCTTGGACGACTTC & \multirow{3}{*}{$\begin{array}{l}\text { Used to sequence verify } \beta \text {-estradiol } \\
\text { inducible expression cassette }\end{array}$} \\
\hline P38 & F & GAGCCTCCGTCGAAGAAAC & \\
\hline P39 & $\mathrm{F}$ & ACGTAAACGGCCACAAGTTC & \\
\hline P40 & $\mathrm{F}$ & AGTCGTCCGGAAACTTGCT & \multirow{4}{*}{$\begin{array}{l}\text { Used to sequence verify DEX inducible } \\
\text { expression cassette }\end{array}$} \\
\hline P41 & $\mathrm{F}$ & CGTTTGGGAGTCGAACACTT & \\
\hline P42 & F & TTCAACCAGTCCGGAAACAT & \\
\hline $\mathrm{P} 43$ & $\mathrm{~F}$ & AGGGCGAGGAGCTGTTCA & \\
\hline $\mathrm{P} 44$ & $\mathrm{~F}$ & GTCCGAACCCTCGACCTC & \multirow{4}{*}{$\begin{array}{l}\text { Used to sequence verify ethanol } \\
\text { inducible expression cassette }\end{array}$} \\
\hline $\mathrm{P} 45$ & F & CGACAAGCTCTTGGAAAACG & \\
\hline P46 & $\mathrm{F}$ & GGACGGTCGTCTTGATTCAC & \\
\hline P47 & F & CACCCTCGTGACCACCTT & \\
\hline P48 & $\mathrm{F}$ & AAACGCCTTGTACGCTTTGT & \multirow{3}{*}{$\begin{array}{l}\text { Used to sequence verify Tetracycline } \\
\text { inducible expression cassette }\end{array}$} \\
\hline P49 & F & CTGGACGGCGACGTAAAC & \\
\hline P50 & $\mathrm{R}$ & GTCCTCCTTGAAGTCGATGC & \\
\hline
\end{tabular}




\begin{tabular}{|c|c|c|c|}
\hline P63 & $\mathrm{F}$ & CGTACCTAGGTCCATTTTGACATGTTTCCTAGCT & \multirow{2}{*}{$\begin{array}{l}\text { Used to clone EF2 Promoter to drive } \\
\text { Transcription factors in the inducible } \\
\text { system }\end{array}$} \\
\hline P64 & $\mathrm{R}$ & CTGAGCGGCCGCCTGTTCAACTGTAAAGTTCACCT & \\
\hline P66 & $\mathrm{F}$ & CGTATCTAGATTTGACGGTTTTTTTTACAAGAGA & \multirow{2}{*}{$\begin{array}{l}\text { Used to clone H4 Promoter to drive } \\
\text { Transcription factors in the inducible } \\
\text { system }\end{array}$} \\
\hline P67 & $\mathrm{R}$ & CTGAGCGGCCGCGTTGGCTGTTGTTTGTTTTCGG & \\
\hline P68 & $\mathrm{F}$ & CGTATCTAGATACGTAGAATCCTACGTAAAATGGA & \multirow{2}{*}{$\begin{array}{l}\text { Used to clone ClP1 Promoter to drive } \\
\text { Transcription factors in the inducible } \\
\text { system }\end{array}$} \\
\hline P69 & $\mathrm{R}$ & CGTAGCGGCCGCTGCGAATGTACGTGTTGTAGC & \\
\hline $\mathrm{P} 222$ & $\mathrm{~F}$ & ACAGCACATGTCATCGGAAA & \multirow{2}{*}{$\begin{array}{l}\text { Used for ddPCR targeting TATA Box } \\
\text { Binding Protein (TBP) reference gene }\end{array}$} \\
\hline $\mathrm{P} 223$ & $\mathrm{R}$ & CATTTGAGCGCGATTTTCTT & \\
\hline P119 & $\mathrm{F}$ & ACAACAGCCACAACGTCTAT & \multirow[t]{2}{*}{ Used for ddPCR targeting eYFP } \\
\hline P120 & $\mathrm{R}$ & TGGTAGCTCAGGTAGTGGTT & \\
\hline $\mathrm{P} 121$ & $\mathrm{R}$ & CTGACCTAGGCTGTTCAACTGTAAAGTTCACCT & PCR amplification of pEF2 \\
\hline $\mathrm{P} 122$ & $\mathrm{R}$ & CTGACCTAGGGTTGGCTGTTGTTTGTTTTCGG & PCR amplification of $\mathrm{pH} 4$ \\
\hline AP1 & $\mathrm{F}$ & CGATTCTAGACGTTACATAACTTACGGTAAATGGC & \multirow[t]{2}{*}{ PCR amplification of pCMVEM7 } \\
\hline AP2 & $\mathrm{R}$ & CGCTCCTAGGGGTTTAGTTCCTCACCTTGTCG & \\
\hline AP4 & $\mathrm{F}$ & CGATTCTAGAACGAATTTCCAACGTCCTAA & \multirow[t]{2}{*}{ PCR amplification of pPt202a } \\
\hline AP5 & $\mathrm{R}$ & CGATCCTAGGGGTGGTGCGGAAAGAGG & \\
\hline AP7 & $\mathrm{F}$ & CAGTTCTAGACATCTTGTTTCTCGTAAGCAAA & \multirow[t]{2}{*}{ PCR amplification of pPt211 } \\
\hline AP8 & $\mathrm{R}$ & CGATCCTAGGGCTATATTTGTTGTGTTCGATTTG & \\
\hline $\begin{array}{l}\text { pJET1. } \\
2\end{array}$ & $\mathrm{~F}$ & CGACTCACTATAGGGAGAGCGGC & \multirow[t]{2}{*}{$\begin{array}{l}\text { Used for sequencing in pJET1.2 } \\
\text { subcloning vector }\end{array}$} \\
\hline $\begin{array}{l}\text { pJET1. } \\
2\end{array}$ & $\mathrm{R}$ & AAGAACATCGATTTTCCATGGCAG & \\
\hline
\end{tabular}



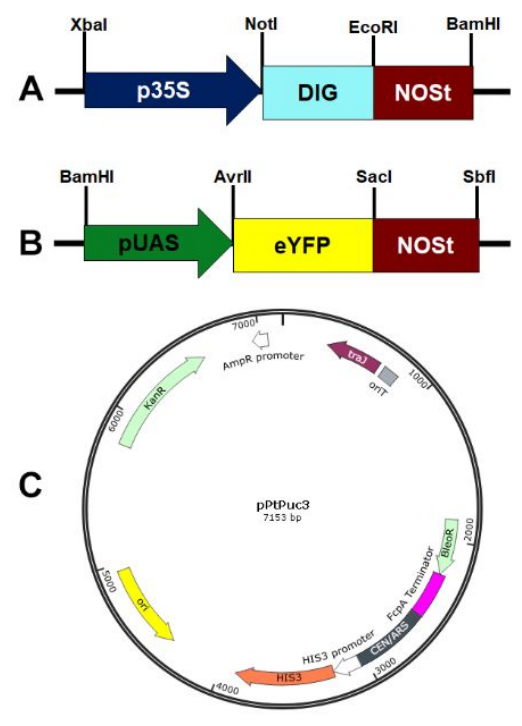

Figure S1. Schematic representation of synthetic gBlock fragments of the DIG expression system and pPtPuc3 episome vector. (A) gBlock fragment 1 of the first transcriptional unit containing p35S promoter driving the chimeric DIG transcription factor. Restriction sites are included to swap genetic components. (B) gBlock fragment 2 of the second transcriptional unit containing pUAS promoter driving eYFP reporter protein. (C) pPtPuc3 episome expression vector map used to assemble all the constructs in this study. All sequences are inserted at the $3^{\prime}$ region of the CEN6-ARSH4-HIS3 sequence using XbaI/SbfI restriction sites based on the recommendation of Karas et.al ${ }^{2}$. 


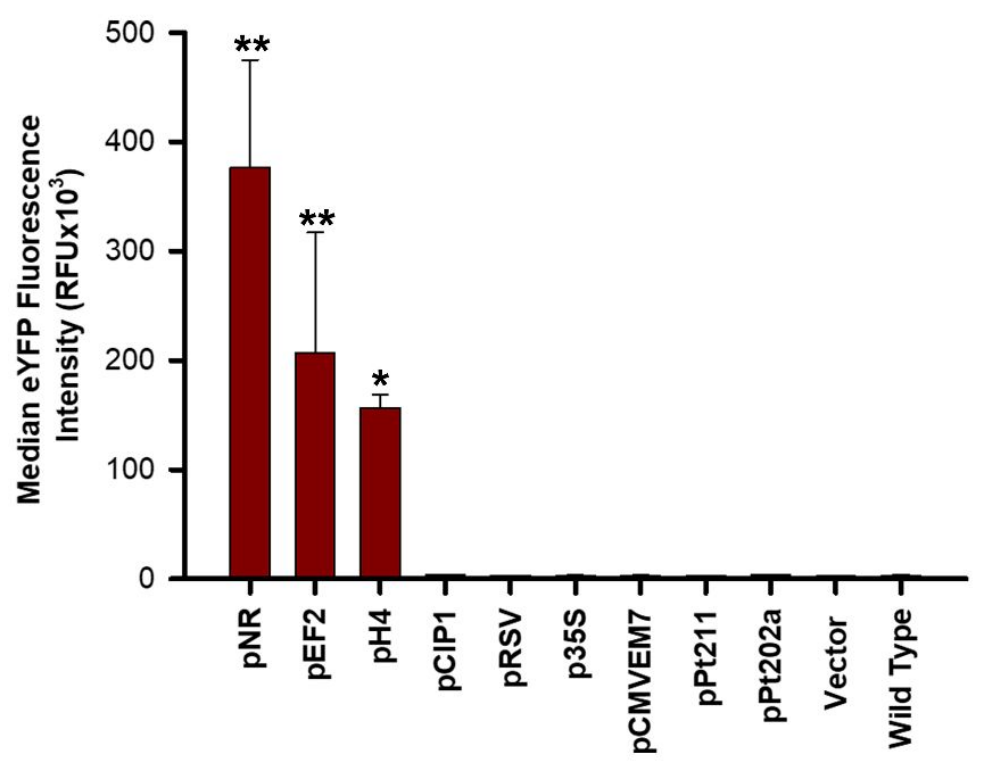

Figure S2. Constitutive promoter characterization. Median fluorescence intensity taken from representative exconjugants of nine constitutive promoters driving eYFP reporter protein. Promoters are of both viral (p35S, pClP1, pRSV, and pCMVEM7) and endogenous (pNR, pEF2, pH4, pPt211, pPt202a) origin. Vector is pPtPuc3 empty vector control and Wild Type is the background autofluorescence intensity. All data are from three biological replicates $(n=3$, mean $\pm \mathrm{sd}$ ). Differences in promoter strength were tested using ANOVA followed by Tukey pairwise analysis. $\mathrm{p}<0.05$ was considered significant. Asterisk $\left(^{*}\right)$ shows a significant difference between groups $(\mathrm{P}<0.01)$. Asterisks $(* *)$ show a significant difference between groups $(\mathrm{P}<0.001)$. 

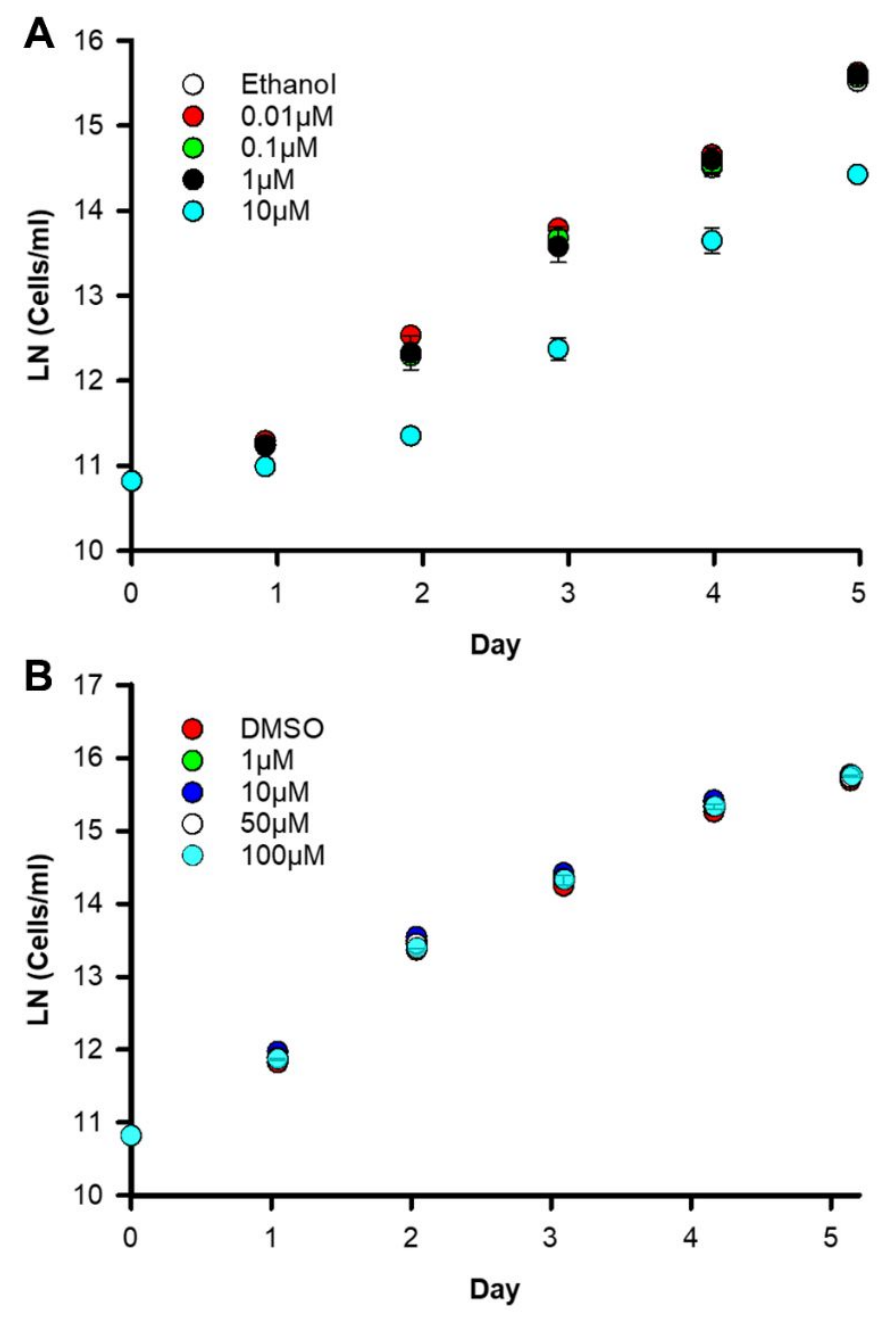

Figure S3. Inducer cytotoxicity assay. Exponentially growing wild-type $P$. tricornutum cells were diluted to $5 \times 10^{5}$ cells $/ \mathrm{ml}$ in $25 \mathrm{ml} \mathrm{f} / 2$ media and grown in the presence or absence of different concentrations of inducer added at time 0 . (A) Test of $\beta$-estradiol concentrations. The solvent control, ethanol, and 0.01, 0.1, 1 and $10 \mu \mathrm{M} \beta$-estradiol were used for this assay. (B) Test of digoxin concentrations. The solvent control, DMSO, and 1, 10, 50 and $100 \mu \mathrm{M}$ digoxin were used for this assay. All data are from three biological replicates $(n=3$, mean $\pm s d)$. 
A

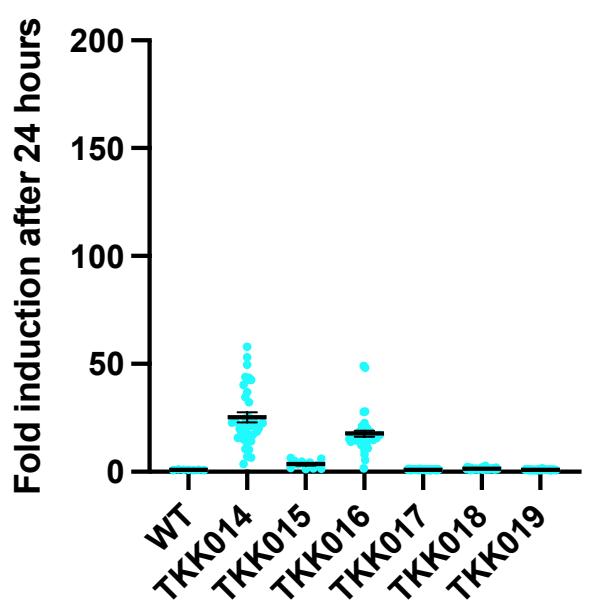

B

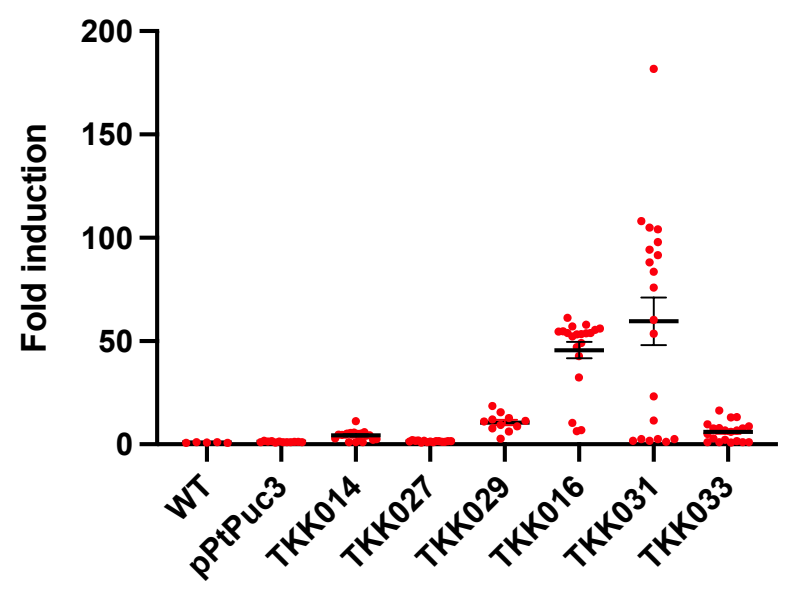

Figure S4. Results of screening transgenic strains for synthetic promoter activity. The fold induction for each strain was calculated by normalizing the mean cellular eYFP fluorescence activation $24 \mathrm{hr}$ after induction to the mean cellular eYFP fluorescence at time zero (before induction). (A) Transgenic strains generated via random integration (electroporation). pNR is used to constitutively express synthetic transcription factors in all inducible systems. WT is wild type control $(n=9)$. TKK014 is the digoxin inducible system $(n=36)$. TKK015 is the 4-OHT inducible system $(n=11)$. TKK016 is the $\beta$-estradiol inducible system $(n=40)$. TKK017 is the dexamethasone inducible system $(n=45)$. TKK018 is the ethanol inducible system $(n=30)$. TKK019 is the tetracycline (Tet-ON) inducible system $(n=33)$. Inducer concentrations of $1 \mu M$ of $\beta$-estradiol, $100 \mu \mathrm{M}$ of digoxin, $10 \mu \mathrm{M}$ 4-hydroxytamoxifon, $10 \mu \mathrm{M}$ dexamethasone, $1 \%$ ethanol, or $1 \mu \mathrm{g} / \mathrm{ml}$ Doxycycline were used. Digoxin inducible strains with fold induction of 49.5, 57.9, and 53,1, and $\beta$-estradiol inducible strains with fold induction of 16.8, 20.8 and 27.9 were selected for further assessment. (B) Transgenic strains generated via conjugation. WT is wild type control $(n=9)$. pPtpuc3 is the empty vector control $(n=6)$. TKK014 is the pNR based digoxin inducible system $(n=20)$. TKK027 is the pEF2 based digoxin inducible system $(n=15)$. TKK029 is the pH4 based digoxin inducible system $(n=11)$. TKK016 is the pNR based $\beta$ - 
estradiol inducible system $(\mathrm{n}=20)$. TKK031 is the $\mathrm{pEF} 2$ based $\beta$-estradiol inducible system $(\mathrm{n}=20)$. TKK033 is the $\mathrm{pH} 4$ based $\beta$-estradiol inducible system $(\mathrm{n}=20)$. The numbers in parentheses are the number of independent strains assayed. Bars show the mean with error bars indicating the standard error of the mean. All data were generated using exponentially growing cells. For induction, $1 \mu \mathrm{M}$ of $\beta$-estradiol was used both constructs. pNR based $\beta$-estradiol inducible strains with fold induction of 58,61.2, 57.1, and pEF2 based $\beta$-estradiol inducible strains with fold induction of 108.1, 104, and 181.7 respectively were used for further assessment in the main text.

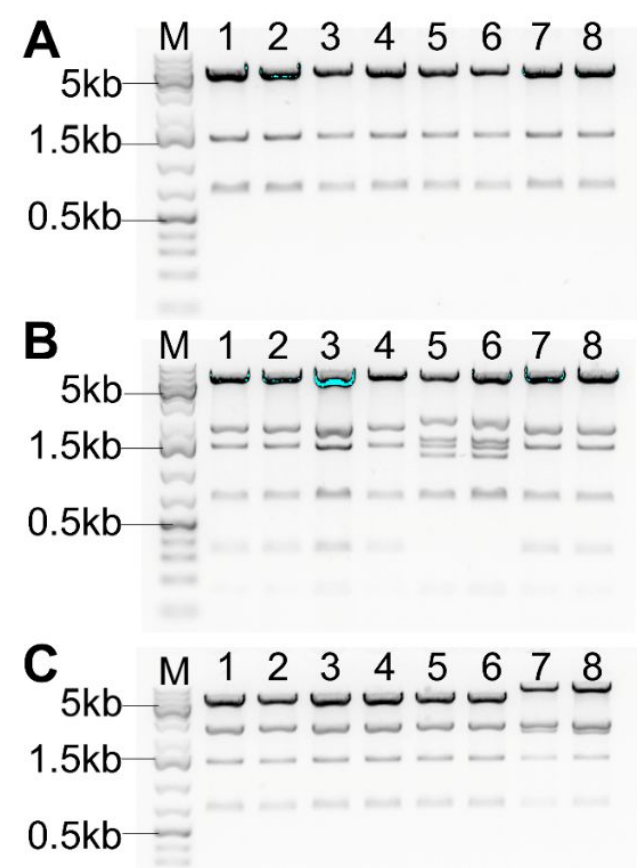

Figure S5. Restriction digestion patterns of episomes rescued from $P$. tricornutum exconjugant lines. (A) Agarose gel electrophoresis showing digestion patterns of episomes rescued from 4 exconjugant lines containing empty vector control, pPtPuc3. (B) Agarose gel electrophoresis showing digestion patterns of episomes rescued from 4 exconjugant lines containing the pNR-based XVE system (TKK016). Cyan color shows band density saturation. (C) Agarose gel electrophoresis showing digestion patterns of episomes rescued from 4 exconjugant lines containing the pEF2-based XVE system (TKK031). BglII and EcoRV restriction enzymes were used for digestion. Duplicate E. coli colonies were selected for each of the exconjugants in this assay. 

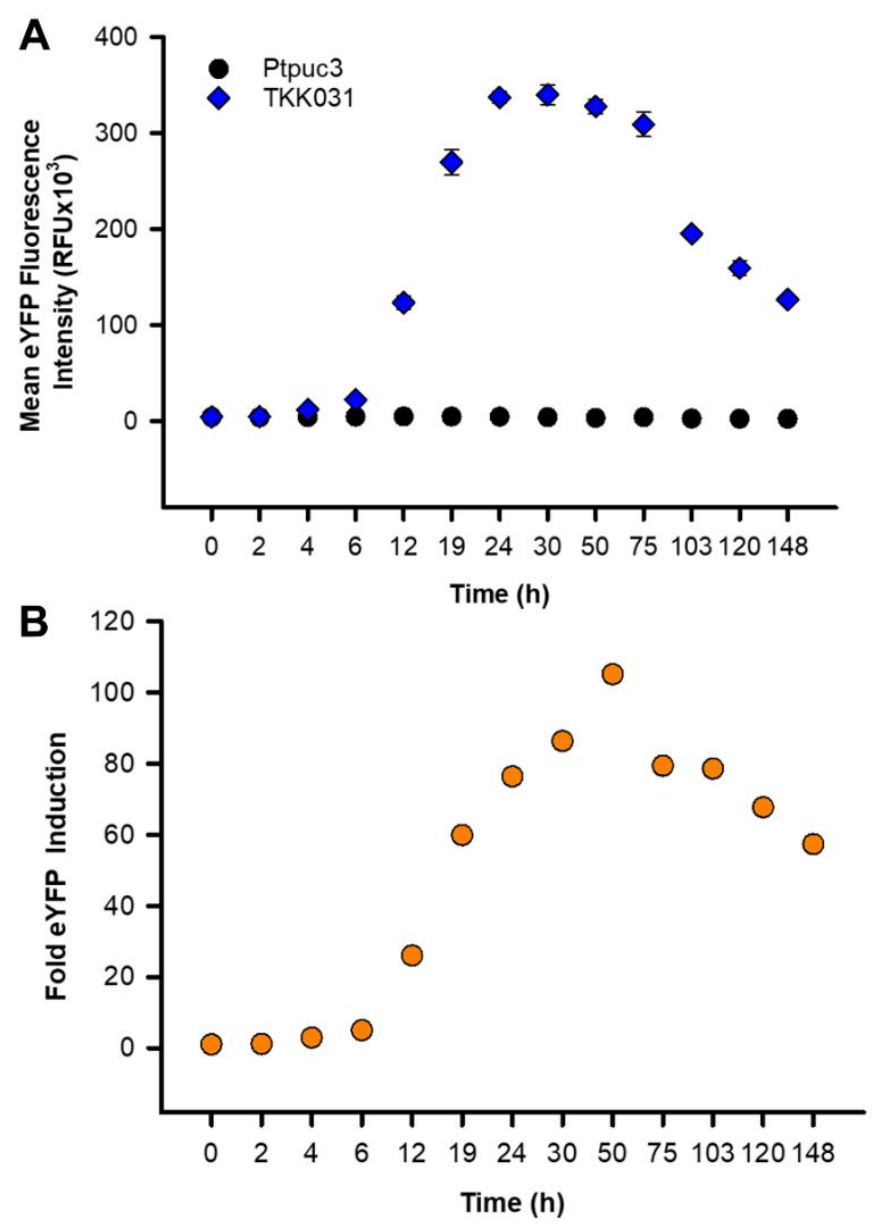

Figure S6. Timecourse induction of pEF2-based $\beta$-estradiol inducible system in $P$.

tricornutum. (A). Mean eYFP fluorescence intensity of exponentially growing $P$. tricornutum cells (starting at 50000 cells $/ \mathrm{ml}$ ) cultured in $\mathrm{f} / 2$ media supplemented one time with $1 \mu \mathrm{M} \beta$ estradiol. The 0 -time point is mean eYFP fluorescence data before induction. PtPuc3 is empty vector control and TKK031 is a pEF2 based $\beta$-estradiol inducible transgenic strain. Error bars shown are the standard deviation of the mean for three independent biological replicates. (B). Fold induction was calculated by normalizing the mean eYFP fluorescence intensity of the transgenic strain (TKK031) at each time point to the corresponding mean eYFP fluorescence of the vector control at each time point. 


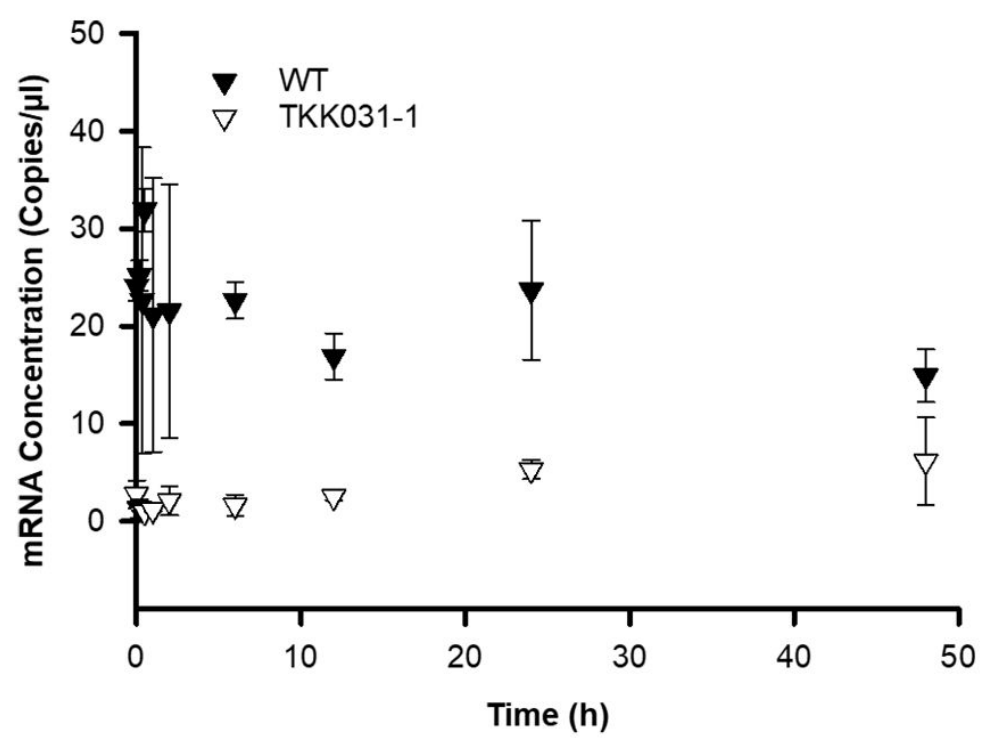

Figure S7. Droplet digital PCR showing no significant induction of the TBP reference gene over time. Undiluted cDNA was used for this assay. WT is wildtype control and TKK031-1 is a representative transgenic strain containing the $\mathrm{pEF} 2$-based $\beta$-estradiol-inducible expression episome generated via conjugation. Samples used are the same as Figure 5. All data are from three biological replicates $(\mathrm{n}=3$, mean $\pm \mathrm{sd})$.

\section{References}

[1] Zou, L.-G., Balamurugan, S., Zhou, T.-B., Chen, J.-W., Li, D.-W., Yang, W.-D., Liu, J.-S., and Li, H.Y. (2019) Potentiation of concurrent expression of lipogenic genes by novel strong promoters in the oleaginous microalga Phaeodactylum tricornutum, Biotechnology and Bioengineering 116, 3006-3015.

[2] Karas, B. J., Diner, R. E., Lefebvre, S. C., McQuaid, J., Phillips, A. P., Noddings, C. M., Brunson, J. K., Valas, R. E., Deerinck, T. J., Jablanovic, J., Gillard, J. T., Beeri, K., Ellisman, M. H., Glass, J. I., Hutchison, C. A., 3rd, Smith, H. O., Venter, J. C., Allen, A. E., Dupont, C. L., and Weyman, P. D. (2015) Designer diatom episomes delivered by bacterial conjugation, Nat Commun 6, 6925. 